\title{
CORRELATION BETWEEN THE PRESENCE OF STROMAL MYOFIBROBLASTS AND THE PROLIFERATIVE POTENTIAL OF ODONTOGENIC KERATOCYST AND AMELOBLASTOMA
}

\author{
Hanan Eid", Naglaa M. Kamal ${ }^{* *}$, Sherif elgayar ${ }^{* * *}$, Amr H. Elbolok ${ }^{* * *}$ and Ahmed Talaat ${ }^{* * * *}$
}

\begin{abstract}
Odontogenic keratocyst (OKC), and ameloblastoma (AB) aggressive epithelial odontogenic tumors with a high recurrence rate though, their aggressive nature are not totally understood, Many epithelial tumors are characterized by stromal reaction, Myofibroblast is one of stromal component that could contribute to the biologic behavior of these lesions, Fifteen cases of $\mathrm{AB}$ and $\mathrm{OKC}$ were operated on under general anesthesia, all cases were subjected to immunohistochemical staining with alpha-smooth muscle actin and flow cytometry analysis, ameloblastoma showed expression of $\alpha$ muscle actin between the follicles as well as around the blood vessels, while in OKC the expression was mostly in connective tissue wall of cyst lining, as well as flow cytometry analysis showed tumors were diploid and showed a percentage of cells in S-phase higher than $26 \%$. OKC had a higher value than $\mathrm{AB}$ but the values were close and statistically insignificant, we concluded that the immunohistochemical expression of MF in $\mathrm{AB} \& \mathrm{OKC}$ has insignificant difference may contribute to the similar proliferative potential of both $\mathrm{AB} \& \mathrm{OKC}$, as well The high proliferation rate of $\mathrm{OKC}$ reinforces its classification as a benign odontogenic neoplasm rather than a cyst
\end{abstract}

Objective To detect the proliferative potential of odontogenic keratocyst, versus ameloblastoma and their correlation to presence of stromal myofibroblasts

\section{INTRODUCTION}

Odontogenic keratocyst (okc), and ameloblastoma (ab) are considered as aggressive epithelial odontogenic tumors with a high recurrence rate though, the pathogeneses of these tumors and their aggressive nature are not totally understood ${ }^{(1)}$.
Ameloblastoma $(\mathrm{Ab})$ is a locally destructive benign epithelial odontogenic neoplasm. It may originate from dental lamina rests, the epithelial lining of an odontogenic cyst, the rests of enamel organ or from the basal cells of the oral mucosa. It comprises about $10 \%$ to $45.2 \%$ of all odontogenic tumors and nearly $1 \%$ of all neoplasms of the oral

* Lecturer of Oral Pathology-Minia University

** Lecturer of Oral Pathology-Ahram/Canadian University

*** Assistant Professor of Oral Pathology-Minia University

**** Lecturer of Oral Maxillofacial Surgery-South Valley University 
cavity. Clinically, Ab arises as a painless swelling with an expansion of the jaw bones. It could be classified into 3 main types: solid or multicystic ameloblastoma, uni-cystic ameloblastoma, and peripheral ameloblastoma. The solid type is the most common type, which has various histopathological patterns as follicular, plexiform, basal cell, granular, acanthomatous and desmoplastic patterns. ${ }^{(2)}$

Odontogenic keratocyst (OKC) is a benign cystic lesion that arises from rests of dental lamina. It is a locally destructive lesion that grows within the medullary spaces of the bone in an anteroposterior direction without causing a notable bony expansion. Due to its aggressive behavior, it was grouped among the benign epithelial odontogenic tumor in $2005^{\cdot(3)}$

Locally aggressive lesions that have high recurrance rate after conventional surgical enucleation, it is well known this is due to the absence of capsule in $\mathrm{AB} \&$ highly friable cyst wall with satellite cysts within okc capsule, the highly aggressive potential of both lesions was attributed to many biological, molecular \& genetic factors in many studies however few studies were done on the correlation between presence of the stromal myofibrblast in both lesions with their invasive potential $^{(4)}$

Myofibroblasts found in the connective tissue stroma and characterized by the presence of a contractile apparatus. They are found in normal tissues such as blood vessels, lymph nodes and bone marrow as well as in various invasive and metastatic malignant tumors. ${ }^{(5)}$.

Many epithelial tumors are characterized by the local accumulation of connective tissue cells and extracellular material; this phenomenon has been called the stromal reaction. One of the cellular components of the stroma reaction is the myofibroblast, a modulated fibroblast that has acquired the capacity to neoexpress alphasmooth muscle actin (actin isoform of vascular smooth muscle cells), and to synthesize important amounts of collagen and other extracellular matrix components. It is now well accepted that the myofibroblast is a key cell for the connective tissue remodeling, which takes place during wound healing and fibrosis development. Myofibroblasts are capable of remodeling connective tissue but also interact with epithelial cells and other connective tissue cells and may thus control such phenomena as tumor invasion and angiogenesis. Few studies have investigated the role of non-epithelial factors such as myofibroblasts (MFs) that could contribute to the biologic behavior of the lesions. So, this study was used to investigate the presence of myofibroblasts in OKC and ameloblastoma and to compare between the presence of stromal myofibroblasts and the biologic aggressiveness of the lesions. ${ }^{(6)}$

A debate regarding the management of these pathologic entities is present in the literature particularly with the presence of several histologic subdivisions and subsequently the pathologic and clinical behavior for each. Treatment options discussed in the literature range from simple procedures like marsupialization or enucleation with curettage to radical resection with discontinuity defects. Radiotherapy was also a choice. Choosing a treatment modality is guided by factors like tumor potential, behavior, physical form plus histologic and growth characteristics, location in the maxillofacial skeleton, tumor size, primary or recurrent growth. More over the patient age and medical condition. ${ }^{(7)}$

Our aim is to correlate between the presence of stromal myofibroblasts in relation to the proliferative potential of odontogenic keratocyst, and ameloblastoma

\section{MATERIAL AND METHODS}

This study was conducted on patients who have been treated for $\mathrm{AB}$ and $\mathrm{OKC}$ in the department of Oral \& Maxillofacial surgery - Faculty of Dentistry/ South Valley University from Jan 2014 to June 2017. 


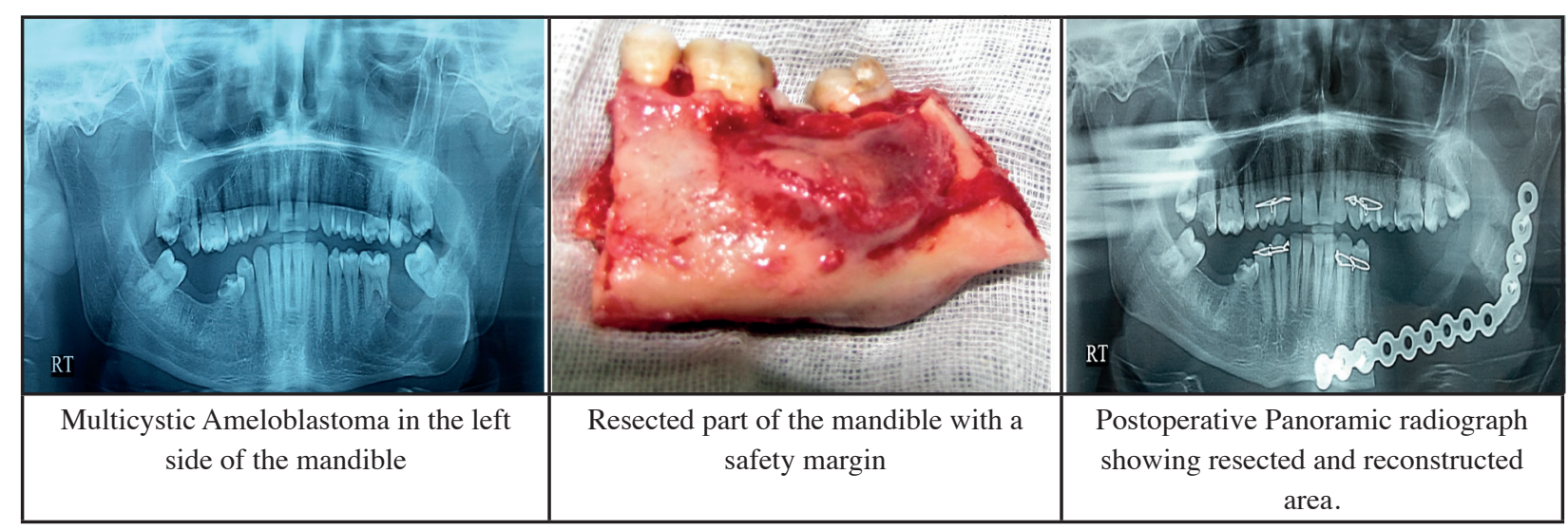

Fifteen cases were operated on under GA. Patients were scrubbed, painted and draped according to the standard surgical protocol. $2 \%$ lignocaine with epinephrine was given at the surgical site. Access for tumors of the anterior segment of the maxilla or the mandible was done via intraoral transmucosal approach. For tumors of the posterior maxilla, a Weber- Ferguson flap was used along with vestibular incision. While for those involving the mandibular body, angle and/or the ramus a combined approach using a standard submandibular and intraoral transmucosal incisions. A resection margin of uninvolved bone of $1.5-2 \mathrm{~cm}$ in solid and multicystic lesions and $0.5-1 \mathrm{~cm}$ for unicystic and peripherl lesions was a standard. The resection margin always lies beyond the tumor invaded tissue plane to assure having a tumor free margin. (Fig.1)

Immunohistochemical study of surgically treated cases of OKC and ameloblastoma, fifteen cases each was selected to make the study. Five-micrometer thick sections were histologically prepared and stained with hematoxylin and eosin stain to confirm the diagnosis under light microscopy.

Three-micrometer thick sections were then obtained from each case for immunohistochemical staining with alpha-smooth muscle actin. The slides were deparaffinized by passing them through two changes of xylene for 5 min each. They were hydrated in two changes of $100 \%$ ethanol for $1 \mathrm{~min}$ each. The slides were then transferred to citrate buffer and autoclaved for antigen retrieval at 15 lbs pressure for $15 \mathrm{~min}$. After cooling, they were washed in phosphate buffer solution. The slides were then treated with protein block reagent for $10 \mathrm{~min}$. Immunohistochemical staining was then performed using $\alpha$ - smooth muscle actin as the manufacturer's instructions. The slides were then mounted in dpx and observed under light microscope for the results.

\section{Flow cytometric analysis}

All steps took place in vacsera (egyptian company for vaccines, sera, and drugs).

Sample preparation: paraffin-embedded specimens were processed for DNA flow cytometric analysis by a technique modified from that of Hedley and co-workers. Sections (thickness, $40 \mathrm{pm}$ ) were deparaffinized in xylene, rehydrated, and subjected to trypsin digestion. After overnight's incubation the nuclear suspension was stained with ethidium bromide $(50 \mathrm{pg} / \mathrm{ml})$, digested with RNAase, and analyzed with an EPICS C flow cytometer (Coulter Electronics, Hialeah, FL) using $488 \mathrm{~nm}$ argon laser excitation.

The criterion for DNA aneuploidy was the presence of two distinct $\mathrm{GO} / \mathrm{Gl}$ peaks. Mean coefficient of variation of the diploid peak was 5.5 (range, 3.2-7.0) which usually allowed detection of DNA peaks with a DNA index greater than 
1.10. Cell cycle analysis was done according to the Baisch rectangular histogram as described previously.'o S-phase was analyzed in such cases where the evaluation was thought to be reliable (89 of 102 tumors). Flow cytometric data analysis was performed by the Coulter Statpak software program (Coulter Electronics, Hialeah, FL).

Analysis of DNA in paraffin-embedded samples was done by isolating cell nuclei from paraffinembedded tissues and used it to retrieve, for flow cytometric analysis. This methodology is useful for studying tumor progression and for assessment of the prognostic significance of DNA ploidy and cell cycle distribution (mainly s-phase cells) (Hedley 1989).

All the results were tabulated and statistically analyzed using computer software named the Statistical Package for Social Science (SPSS version 16). Data in the present study were presented as mean and standard deviation (SD). The comparisons between the study groups were determined by using an independent-samples $t$ test and paired $t$ - test. The level of significance was established at the value of $\mathrm{P}<0.05$.

\section{RESULTS}

A total number of thirty cases were taken for the study, fifteen from each group. All cases showed positive immunoreaction in the stroma for $\alpha$ smooth muscle actin. The ameloblastoma showed expression of $\alpha$ muscle actin between the follicles as well as around the blood vessels (Fig. 2), while in OKC the expression was mostly in c.t wall of cyst lining (Fig. 3)

\section{Image Analysis Results}

Mean values of area fraction of smooth muscle actin immunopositivity in odontogenic keratocyst, and ameloblastoma was (4.8825 \& 4.4819) respectively

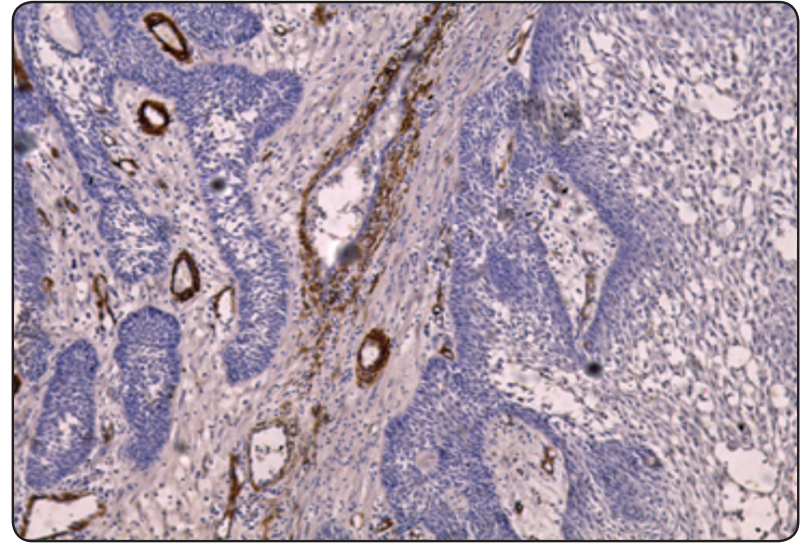

Fig. (2) Photomicrograph showing the expression of $\alpha$ smooth muscle actin in multicystic $\mathrm{AB}$ showing immunostaining in the stroma between follicles as well as around blood vessels. (X100)

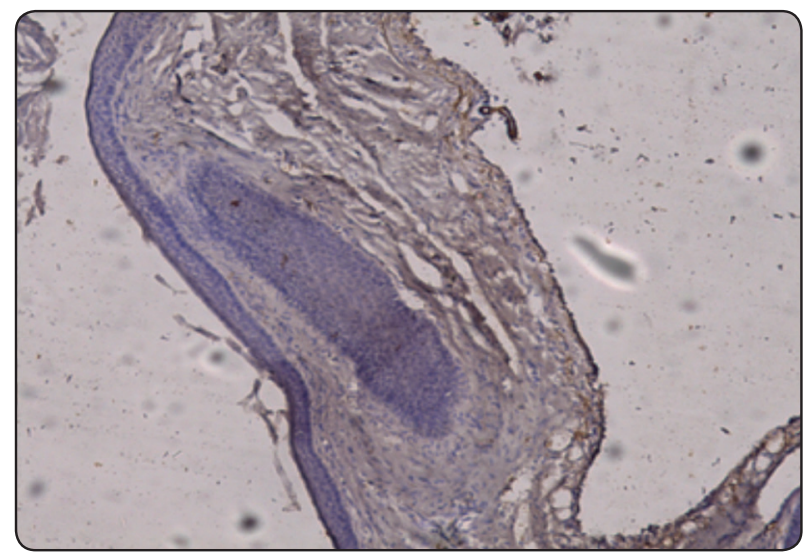

Fig. (3) Photomicrograph showing the expression of $\alpha$ smooth muscle actin in OKC showing immunostaining in the connective tissue wall of cyst lining. (X40)

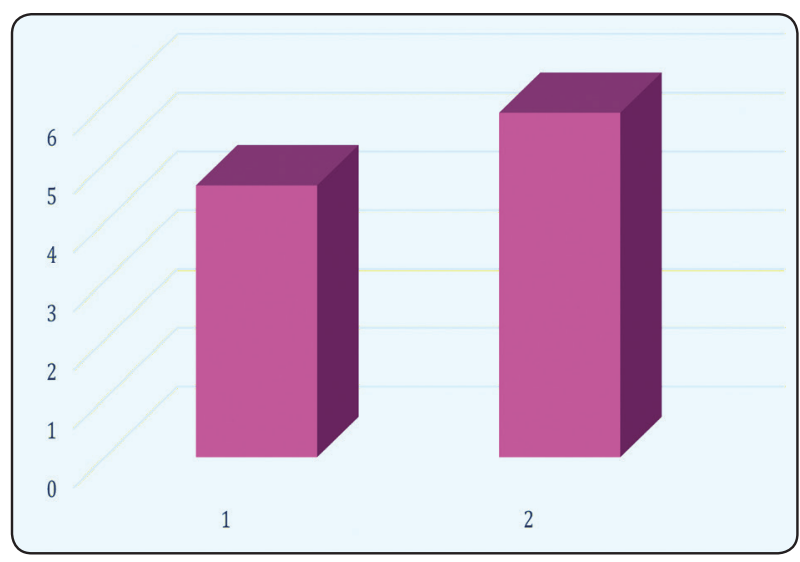

Fig. (4): A histogram representing the mean area fraction of smooth muscle actin immunopositivity in ameloblastoma (1), and odontogenic keratocyst (2). 


\section{Statistical results}

The stastical results showed an insignificant correlation between $\mathrm{AB} \& \mathrm{OKC}$ expression of smooth muscle actin $(\mathrm{P}=0.541)$ table (1)

\section{Flow cytometric results}

All the examined tumors were diploid and showed a percentage of cells in $\mathrm{S}$-phase higher than $26 \%$. OKC had a higher value than $\mathrm{AB}$ but the values were close and statistically insignificant. (fig 5)
TABLE (1) T-test for equality of means for smooth muscle actin in odontogenic keratocyst, and ameloblastoma.

\begin{tabular}{|c|c|c|c|c|}
\hline Group & $\mathrm{N}$ & Mean & $\begin{array}{c}\text { Std. } \\
\text { deviation }\end{array}$ & $\mathrm{P}$ value \\
\hline $\begin{array}{ll}\text { Area } & \mathrm{AB} \\
\text { fraction \% } & \mathrm{KCOT}\end{array}$ & $\begin{array}{l}15 \\
15\end{array}$ & $\begin{array}{l}4.4819 \\
4.8825\end{array}$ & 2.51069 & 0.541 \\
\hline
\end{tabular}

\section{Significant less than 0.05}
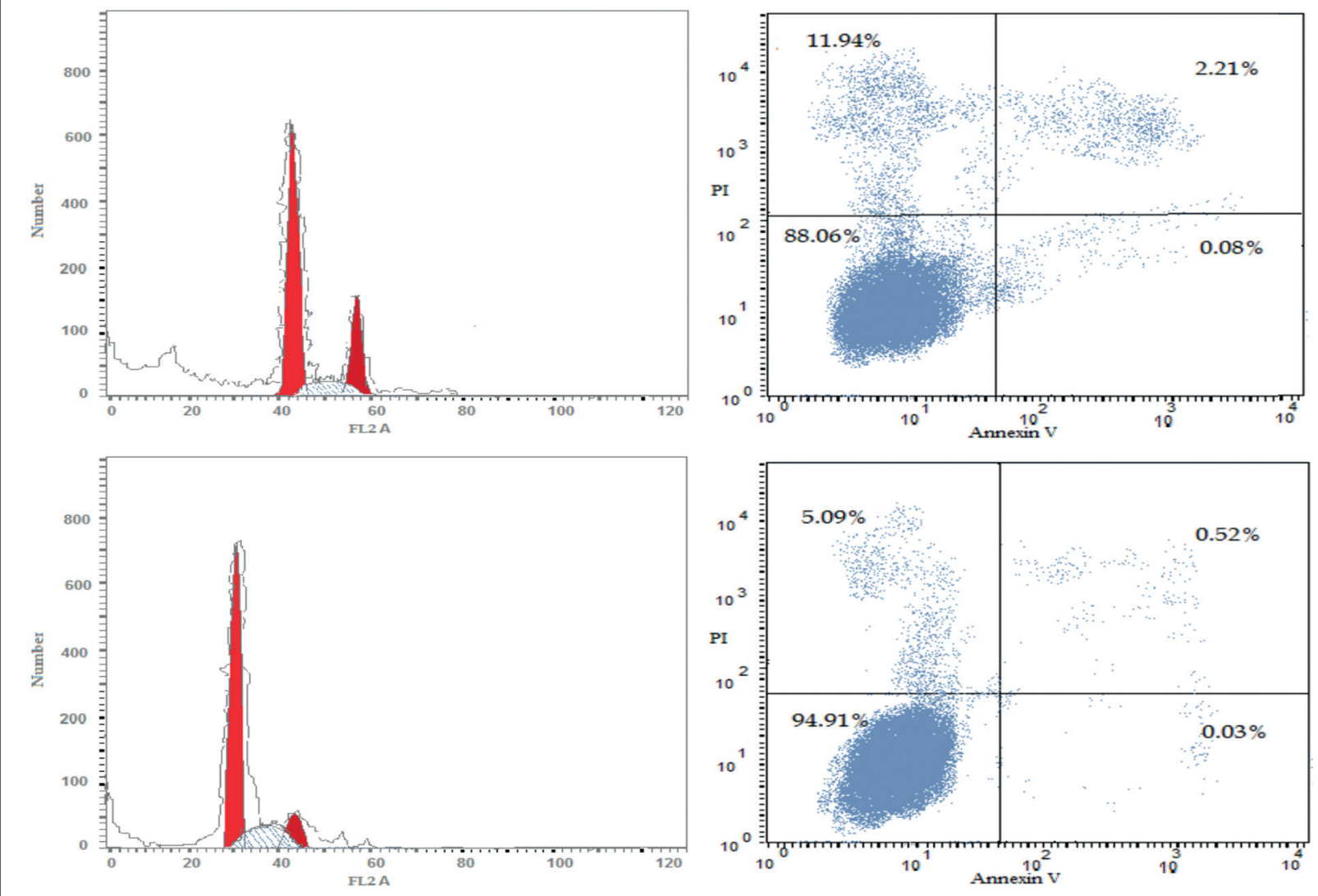

Fig. (5) 


\section{DISCUSSION}

The neoplastic cells lead to abnormalities within the epithelium due to which the stroma changed to reactive one, such neoplastic cells secrete TGF- $\beta 1$ cytokine which leads to the differentiation of fibroblasts into $\mathrm{MFs}^{\cdot}{ }^{(8)}$. The myofibroblasts release cytokines and matrix metalloproteases which destruct the ECM leading to tumor growth, invasion and metastasis ${ }^{\cdot(9)}$

In the present study we investigate the presence, and area fraction of MFs using immunohistochemical marker, which is $\alpha$-SMA in two odontogenic lesions, which are OKC and ameloblastoma, and to correlate the results with the aggressiveness of the lesions by flow cytometry.

In our study the area fraction percentage of MFs were slightly higher in $\mathrm{OKC}$ than $\mathrm{AB}$ but without any statistically significant difference. This was in accordance with another study by Vered et al in $2005^{(10)}$ states that OKC showed the lowest number of MF positivity among the odontogenic cysts studied, whereas OKC showed the highest MF positivity.

Two other studies ${ }^{(11,12)}$ said that MF positivity was higher in OKC than in ameloblastoma, which was also in accordance with our results.

In contrary to our results, Deepa et al $2016^{(13)}$ said that ameloblastoma showed a marginally increased count of MF when compared to OKC, but this difference too was not significant.

Allison and Spencer ${ }^{(14)}$ were shown that the mean silver stained nucleolar-organizing regions (AgNORs) count was highest in ameloblastoma, followed closely by OKC. AgNOR, was a measure of the clinical behavior of the lesions and those with higher scores would be expected to exhibit a more aggressive biologic behavior.

Lombardi and Morgan ${ }^{(15)}$ confirm the presence of the MFs in the wall of odontogenic cysts and suggested that MFs may be part of a homeostatic response to the distension of the cyst wall caused by the cyst enlargement. So, increased number of MFs in the stroma of OKC may be considered as directly proportional to its reported aggressive behavior.

The presence of MFs in the stroma of SCC especially at the invasive margins was related to the role of these cells in the growth and progression of the lesion, by their ability to alter the ECM. The increased density of stromal MFs in poorly differentiated oral SCCs has been related to the involvement of MFs in the creation of a permissive microenvironment in the stroma for tumor growth, progression and invasion. ${ }^{(16)}$

The presence of MFs in the stroma just beneath the lining epithelium in $\mathrm{OKC}$ and surrounding the tumor islands in ameloblastoma, in our study, may be an indicator to the role of these cells in the growth and further progression of these lesions.

Our immunohistochemical results were in accordance with flow cytometrical results which revealed in the present study an increase in the percentage of cells in $\mathrm{S}$ phase in all the studied cases (all were above 26\%).

Within the cell cycle, cell nuclei contain different amounts of DNA according to the stage of the cycle. Upon receiving proliferation signals, diploid cells exit its resting state Gap 0 phase and enter the Gap 1 phase. At Gap 1 phase, the diploid cells maintain their ploidy (having two complete sets of chromosomes) (2N). Replication of DNA starts when the cells enter the synthesis phase. In this phase, cells contain different amounts of DNA. Replication of DNA persists until the DNA content is twice that of the diploid state and be in a tetraploid state $(4 \mathrm{~N})$. These tetraploid cells in the Gap2 phase prepare for the division and enter the mitosis phase in which the cells divide into two identical diploid $(2 \mathrm{~N})$ cells. The daughter cells may enter another division cycle or enter the resting stage. Based only on DNA content, the M phase is identical to the G2 phase, and G0 is identical to G1. 
So, the cell cycle is commonly described by the G0/G1, S, and G2/M phases when based on DNA content ${ }^{(17) \text {. }}$

During apoptosis, extensive fragmentation DNA occurs. The apoptotic cells are defined as "sub-G1" cells. ${ }^{(18,19)}$

The present study revealed an increase in the percentage of cells in $\mathrm{S}$ phase in all the studied cases (all were above 26\%). The study of Ostovic et al, $2017^{(20)}$ revealed an increase in the percentage of cells of AB in the S-phase (25.9\%) they consider this percentage as an indication of an unregulated, high proliferation.

Treatment of $\mathrm{OKC}$ and $\mathrm{AB}$ is a major challenge for surgeons. Many surgical techniques have been proposed aiming for complete removal and minimize recurrent or residual disease. Unfortunately, there is no literature consensus on a standard management protocol as each of these protocols have their proponents and support to varying degrees in the literature. Surgical techniques employed include: enucleation and curettage, enucleation and chemical fixation, enucleation and cryotherapy, enucleation and peripheral osteotomy, marsupialization and resection. Frequent results reported in the literature after the usage of each technique or after comparing techniques together guided its use.

Enucleation and curettage is considered the most conservative treatment option for $\mathrm{OKC}$ and $\mathrm{AB}$. However, recurrence rates as high as $62.5 \%$ and $100 \%$ have been reported for OKCs and $\mathrm{AB}$ correspondingly. Consequently it is not recommended by most authors. ${ }^{(21,22)}$

We used resection technique for managing all cases enrolled in this study. Resection is the only predictably curative procedure for patients with $\mathrm{OKC}$ and $\mathrm{AB}$ that offers a recurrence rate approaching zero.

We applied a resection margin of uninvolved bone of $1.5-2 \mathrm{~cm}$ in solid and multicystic lesions and $0.5-1 \mathrm{~cm}$ for unicystic and peripherl lesions to assure having a tumor free margins. This was proved by our histopathologic results. Tumor resection may be completed in the form of composite, segmental, or marginal. The drawn results of different research work regarding high recurrence after enucleation and curettage and better results after resection in $\mathrm{OKC}$ and $\mathrm{AB}$ confirm the findings of this research as the proliferating growth pattern of keratotic odontogenic tumor and ameloblastoma could be considered as the pathological base of their local aggressive behavior.

\section{CONCLUSION}

The immunohistochemical expression of MF in $\mathrm{AB} \& \mathrm{OKC}$ has insignificant difference may contribute to the similar proliferative potential of both $\mathrm{AB} \& \mathrm{OKC}$.

The high proliferation rate of $\mathrm{OKC}$ reinforces its classification as a benign odontogenic neoplasm rather than a cyst.

\section{REFERENCES}

1- Diana H., Maya R., Sekar B., Murali S., Ramesh K. "Expression of inducible nitric oxide synthase in keratocystic odontogenic tumor and variants of ameloblastoma-A comparative study.” JCDR. 2014; 8:115.

2- Rapidisa A.D., Andressakisa S.D., Stavrianosa G.F., aratzisa N., Arnogiannaki-Liappib G.A.L., agogiannisa S.V.V., alsamisa N., Apostolikas." Ameloblastomas of the jaws: clinico-pathological review of 11 patients." European Journal of surgical Oncology. 2004; Vol. 30, Iss 9.

3- Amaral F.R., Paixão G.C., Bonisson L.A., Mesquita R.A., Rebello M.C.; Helenice de Andrade Marigo. "Cell proliferation and apoptosis in ameloblastomas and keratocystic odontogenic tumors.” Brasilian Dental Journal. 2012; vol. 23 no. 2

4- Telles D.C., Castro W.H., Gomez R.S., Souto G.R., Mesquita R.A." Morphometric evaluation of keratocystic odontogenic tumor before and after marsupialization." Braz. oral res. 2013; vol.27.

5- Otranto M., Sarrazy V., Bonte F., Hinz B. Gabbani G., Desmouliere A.," The role of the myofibroblast in tumor stroma remodeling." Cell Adh Migr. 2012; 1; 6(3)203-219. 
6- Desmouliere A., Guyot C., Gabbini G.," The stroma reaction myofibroblast: a key player in the control of tumor cell behavior.” Int J Deve Biol. 2004; 48(5-6); 509-17.

7- Fonseca R.J., Marciani R.D., Turvey T.A., "Oral and Maxillofacial Surgery. St. Louis, Mo.: Saunders/Elsevier, 2009. 3 v. 29 cm. Edición ; 2nd ed.

8- Lewis MP., Lygoe KA., Nystrom ML., Anderson WP., Speight PM., Marshall JF., et al. "Tumour-derived TGFbeta1 modulates myofibroblast differentiation and promotes HGF/SF-dependent invasion of squamous carcinoma cells.” Br J Cancer. 2004; 90:822-32.

9- De Wever O., Mareel M., "Role of tissue stroma in cancer cell invasion.” J Pathol. 2003; 200:429-47.

10- Vered M., Shohat I., Buchner A., Dayan D., "Myofibroblasts in stroma of odontogenic cysts and tumors can contribute to variations in the biological behavior of lesions." Oral Oncol. 2005; 41:1028-33.

11- Mashhadiabbas F., Atarbashi MS., Moshref M., Elahi M.,' Immunohistochemical detection and ultrastructure of myofibroblasts in the stroma of odontogenic cysts and ameloblastoma.” Iran Red Crescent Med J. 2010; 12:453-7.

12- Joshi P., Bhosale S., Hongal B., Chougule M., Dudanakar M.," Comparison of immunoexpression of $\alpha$-SMA in inflamed and non-inflamed odontogenic keratocyst and ameloblastoma.” Int J Appl Dent Sci. 2014; 1:4-9.

13- Deepa Syamala., Rakesh Suresh., Mahija Janardhanan., Vindhya Savithri., Prem P Anand., and Amrutha Jose1. "Immunohistochemical evaluation of myofibroblasts in odontogenic cysts and tumors: A comparative study." J Oral Maxillofac Pathol. 2016; May-Aug; 20(2): 208-213.

14- Allison RT., Spencer S. "Nucleolar organiser regions in odontogenic cysts and ameloblastomas.” Br J Biomed Sci. 1993; 50:309-12.
15- Lombardi T., Morgan PR. "Immunohistochemical characterisation of odontogenic cysts with mesenchymal and myofilament markers." J Oral Pathol Med. 1995; 24:170-6.

16- De-Assis EM., Pimenta LG., Costa-e-Silva E., Souza PE., Horta MC. "Stromal myofibroblasts in oral leukoplakia and oral squamous cell carcinoma."Med Oral Patol Oral Cir Bucal. 2012; 17:733-8.

17- Kapinas K., Grandy R., Ghule P., Medina R., Becker K., PARDEE A, et al. "The abbreviated pluripotent cell cycle." J Cell Physiol. 2013; 228:9-20.

18- Kajstura M., Halicka HD., Pryjma J., et al. "Discontinuous fragmentation of nuclear DNA during apoptosis revealed by discrete "sub-G1" peaks on DNA content histograms." Cytometry A. 2007; 71A:125-31.

19- Darzynkiewicz Z., Huang X., Okafuji M., King MA. “Cytometric methods to detect apoptosis." Methods Cell Biol. 2004; 75:307-341.

20- Karmen Trutin Ostovic1., Berislav Rožman2., Ivica Lukšić3., Mišo Virag3., Dalibor Frančeski4., Zorana Lipšanski1 and Spomenka Manojlović5. "Cytology and DNA analysis of ameloblastma-Acase report." Hygiene Science. 2017; 2573-7717.

21- Zhao Y., Wei JZ., Wang WP. "Treatment of odontogenic keratocysts: a follow-up of 255 Chinese patients." Oral Surg Oral Med Oral Pathol Oral Radiol Endod 2002; 94:151-6.

22- Lionel Gold \& Thomas P. Williams: "Odontogenic Tumors: Surgical Pathology and Management. In Fonseca, Marciani and Turvey, editors. Oral and Maxillofacial Surgery. $2^{\text {nd }}$ ed. Saunders Elsiever, USA, pp 499, 2009), (Carlson ER \& Marx RE: The Ameloblastoma: primary, curat ive surgical management. J Oral Maxillofac Surg 64: 484, 006 